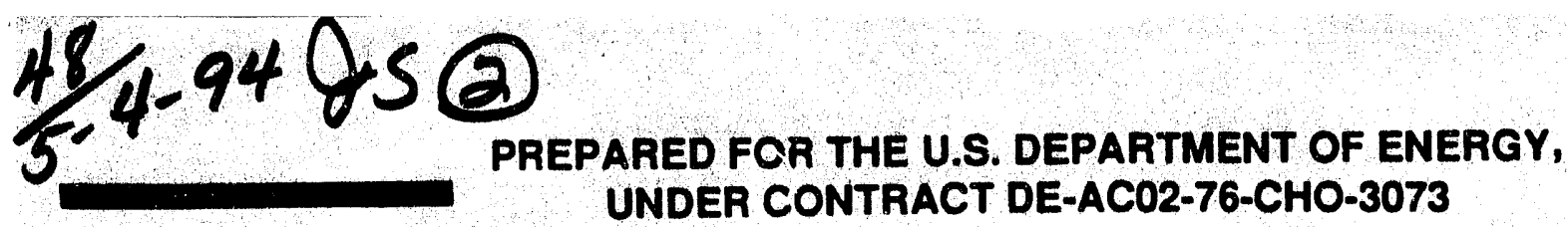

PPPL-2984

UC-427

SELF-CONSISTENT STUDY OF THE ALPHA PARTICLE DRIVEN TAE MODE

BY

Y. WU AND R.B. WHITE

APRIL, 1994
PPPL-2984 


\section{NOTICE}

This report was prepared as an account of work sponsored by an agency of the United States Government. Neither the United States Government nor any agency thereof, nor any of their employees, makes any warranty, express or implied, or assumes any legal liability or responsibility for the accuracy, completeness, or usefulness of any information, apparatus, product, or process disclosed, or represents that its use would not infringe privately owned rights. Reference herein to any specific commercial produce, process, or service by trade name, trademark, manufacturer, or otherwise, does not necessarily constitute or imply its endorsement, recommendation, or favoring by the United States Government or any agency thereof. The views and opinions of authors expressed herein do not necessarily state or reflect those of the United States Government or any agency thereof.

\section{NOTICE}

This report has been reproduced from the best available copy. Available in paper copy and microfiche.

Number of pages in this report: 29

DOE and DOE contractors can obtain copies of this report from:

Office of Scientific and Technical Information P.O. Box 62

Oak Ridge, TN 37831;

(615) $576-8401$.

This report is publicly available from the:

National Technical Information Service

Department of Commerce 5285 Port Royal Road

Springfield, Virginia 22161

(703) $487-4650$ 


\title{
Self-Consistent Study of the Alpha Particle Driven TAE Mode
}

\author{
Yanlin Wu and Roscoe B. White \\ Plasma Physics Laboratory, Princeton University \\ P. O. Box 451 \\ Princeton, New Jersey 08540 USA
}

\begin{abstract}
The interaction of high energy particles with an Alfven eigenmode is investigated selfconsistently by using a realistic kinetic dispersion relation. All important poloidal mode numbers and their radial mode profiles as calculated with the NOVA-K code are included. A Hamiltonian guiding center code is used to simulate the alpha particle motion. The numerical simulations include particle orbit width, nonlinear particle dynamics and the effects of the modes on the particles. Modification of the particle distribution leading to mode saturation is observed. Particle loss is limited to devices in which the alpha particle gyro radius is a significant fraction of the minor radius.
\end{abstract}




\section{Introduction}

In recent years considerable progress has been made in the development of fusion energy, with tokamak experiments rapidly approaching ignition conditions. To sustain ignition in a burning plasma, it is crucial that most of the alphas produced in deuterium-tritium fusion reactions must be confined long enough to heat the bulk plasma. Collective alpha-driven instabilities such as toroidicity-induced Alfven eigenmodes (TAE) are of concern for future tokamak devices since they can induce anomalous alpha losses. ${ }^{1-7}$

In this paper, we study the alpha-particle driven TAE instability numerically by following the evolving alpha particle distribution and solving the kinetic dispersion relation, giving the instantaneous mode frequency and growth rate. The simulations include the full nonlinear particle dynamics, finite orbit width and the effects of the modes on the particles.

\section{Dispersion Relation}

We consider an axisymmetric toroidal plasma consisting of a core plasma and a hot ion species. The core plasma is described by ideal magnetohydrodynamics (MHD) and the hot component by the gyro-kinetic equation. The ion- and electron-Landau damping, collisional damping and continuum damping are taken as parameters supplied by other analysis. The linearized momentum equation is

$$
\omega^{2} \rho \xi=\nabla \delta P_{c}+\nabla \cdot \delta \mathbf{P}_{h}+\mathbf{B} \times(\nabla \times \delta \mathbf{B})+\delta \mathbf{B} \times(\nabla \times \mathbf{B}),
$$

where $\xi$ is the usual fluid displacement vector, $\omega$ is the mode frequency, $\rho$ is the fluid density, $\delta P_{c}$ is the perturbed fluid presure, which is assumed isotropic, $\delta \mathbf{P}_{h}$ is the perturbed hot component pressure tensor, and $\mathbf{B}$ and $\delta \mathbf{B}_{\perp}$ are the equilibrium and the perturbed magnetic 
field, respectively. The following ideal MHD relations hold for the shear Alfven wave:

$$
\begin{array}{cc}
\delta \mathbf{B}_{\|}=0 & \delta \mathbf{B}_{\perp}=\nabla \times\left(\xi_{\perp} \times \mathbf{B}\right) \\
\delta \mathbf{E}_{\|}=0 & \delta \mathbf{E}_{\perp}=i \omega\left(\xi_{\perp} \times \mathbf{B}\right)
\end{array}
$$

The perturbed pressure tensor can be written in a diagonal form,

$$
\begin{gathered}
\nabla \delta P_{c}=-\xi_{\perp} \cdot \nabla P_{c} \\
\delta \mathbf{P}_{h}=\delta P_{\perp} \mathbf{I}+\left(\delta P_{\|}-\delta P_{\perp}\right) \mathbf{b b}
\end{gathered}
$$

where the equilibrium is assumed to be isotropic, and $\delta P_{\|}$and $\delta P_{\perp}$ are obtained from the perturbed particle distribution function $\delta f$ through

$$
\left(\begin{array}{c}
\delta P_{\perp} \\
\delta P_{\|}
\end{array}\right)=\int d^{3} \mathbf{v} \delta f\left(\begin{array}{c}
\mu B \\
2(E-\mu B)
\end{array}\right)
$$

where $E$ is the particle energy, $\mu$ is the magnetic moment, and $\delta f$ has been derived previously. ${ }^{8,9}$

Write the perturbed distribution function $\delta f$ as:

$$
\delta f=\frac{e_{\alpha}}{m_{\alpha}} \partial_{E} F \Phi+\delta g
$$

where $e_{\alpha}$ is the alpha particle charge, $m_{\alpha}$ is the mass, $F$ is the equilibrium distribution function, $\Phi$ is the electrostatic potential, and $\delta g$ is determined by the drift-kinetic equation. With $\delta g \sim e^{i n \phi-i \omega t}$, we have

$$
\left(-i \omega+v_{\|} \mathbf{b} \cdot \nabla+\mathbf{v}_{d} \cdot \nabla\right) \delta g=-i \frac{e_{\alpha}}{m_{\alpha} E} F\left(\bar{\omega}-\omega_{*}\right)\left(\Phi-v_{\|} A_{\|} / c\right)
$$

where $\bar{\omega}=-(\omega E) \partial_{E} \log F$ and $\omega_{*}=-\left(n E / e_{\alpha}\right) \partial_{\psi_{p}} \log F$ and $\psi_{p}$ is the poloidal flux. We have used axisymmetry to restrict consideration to a single toroidal mode number $n$. 
Let

$$
\delta g=\frac{e_{\alpha}}{m_{\alpha} E} F\left(\bar{\omega}-\omega_{*}\right) \Phi+\hat{g} e^{-i \omega t}
$$

giving for the total perturbed particle distribution:

$$
\delta f=-\xi_{\perp} \cdot \nabla F+\hat{g}
$$

The first term on the right-hand side is the adiabatic response and the second term is the non-adiabatic part.

The non-adiabatic particle distribution $\hat{g}$ satisfies the equation

$$
\left(-i \omega+v_{\|} \mathbf{b} \cdot \nabla+\mathbf{v}_{d} \cdot \nabla\right) \hat{g}=-i 2 F\left(\bar{\omega}-\omega_{*}\right) J\left(\psi_{p}, \theta, \phi\right)
$$

where

$$
J\left(\psi_{p}, \theta, \phi\right)=\frac{\epsilon_{\alpha}}{2 m_{\alpha} E c} v_{\|} A_{\|}=\left(1-\frac{3 \mu B}{2 E}\right) \kappa \cdot \xi_{\perp}
$$

and $\kappa$ is the magnetic field line curvature.

We are interested in the resonant response at Alfven frequencies. Only passing particle transit frequencies are large enough to contribute. Thus to solve Eq. (11), we first let $v_{\|}=\left\langle v_{\|}\right\rangle+\tilde{v}_{\|}$, where $\langle\cdots\rangle$ means the orbit average. Since $\left\langle v_{\|}\right\rangle=0$ for trapped particles, the dominant contribution to $\hat{g}$ is from the passing population. Represent the function $J\left(\psi_{p}, \theta, \phi\right)$ by a Fourier series in $\theta$,

$$
J\left(\psi_{p}, \theta, \phi\right)=\sum_{l} J_{l}\left(\psi_{p}\right) e^{i S_{l}}
$$

where $S_{l}=n \phi-l \theta$.

To lowest order, let

$$
\hat{g}\left(\psi_{p}, \theta, \phi\right)=\sum_{l} g_{l}\left(\psi_{p}\right) e^{i S_{l}}
$$


In the high frequency limit, $\omega \sim \omega_{t} \gg \omega_{b}$, with $\omega_{t}, \omega_{b}$ the transit frequency and the bounce frequency respectively, we find

$$
g_{l}\left(\psi_{p}\right)=2 F\left(\omega_{*}-\bar{\omega}\right) \frac{J_{l}\left(\psi_{p}\right)}{k_{l}\left\langle v_{\|}\right\rangle-\omega}
$$

with

$$
k_{l}=\frac{n q-l}{q R}
$$

The final form of $\hat{g}$ is then

$$
\hat{g}\left(\psi_{p}, \theta, \phi\right)=2 F\left(\bar{\omega}-\omega_{*}\right) \sum_{l} \frac{J_{l}}{k_{l}\left\langle v_{\|}\right\rangle-\omega} e^{i S_{l}} .
$$

We now derive the dispersion relation variationally. Perform $\int \xi^{*} \cdot$ on Eq. (1) and assume a fixed conducting boundary. We then obtain the dispersion relation:

$$
D(\omega)=\delta K+\delta W_{f}+\delta W_{k}^{(a)}+\delta W_{k}^{(n a)}=0
$$

where

$$
\delta K=-\frac{1}{2} \omega^{2} \int \rho|\xi|^{2} d^{3} x
$$

is the kinetic energy and

$$
\begin{gathered}
\delta W_{f}=\frac{1}{2} \int d^{3} \mathbf{x}\left\{\left|\delta \mathbf{B}_{\perp}\right|^{2}+\left|2 \kappa \cdot \xi_{\perp}\right|^{2} B^{2}\right. \\
\left.+\left(\mathbf{J} \cdot \mathbf{B} / B^{2}\right)\left(\delta \mathbf{B}_{\perp} \times \mathbf{B}\right) \cdot \xi_{\perp}^{*}-2\left(\kappa \cdot \xi_{\perp}^{*}\right)\left(\nabla P_{c} \cdot \xi_{\perp}\right)\right\}
\end{gathered}
$$

is the potential energy due to the core plasma. The high energy contributions are

$$
\delta W_{k}^{(a)}=-\int d^{3} \mathbf{x}\left(\kappa \cdot \xi_{\perp}^{*}\right)\left(\nabla P_{h} \cdot \xi_{\perp}\right)
$$

which is the adiabatic part of the energetic particle potential energy, and

$$
\delta W_{k}^{(n a)}=-\frac{1}{2} \int d^{3} \mathbf{x}\left(\delta \hat{p}_{\perp}-\delta \hat{p}_{\|}\right)\left(\kappa \cdot \xi_{\perp}^{*}\right)
$$


which is the non-adiabatic part of the energetic particle potential energy. The perturbed pressure due to the non-adiabatic part is given by

$$
\left(\begin{array}{c}
\delta \hat{p}_{\perp} \\
\delta \hat{p}_{\|}
\end{array}\right)=\int d^{3} v \hat{g}\left(\begin{array}{c}
\mu B \\
2(E-\mu B)
\end{array}\right) .
$$

Using the $\hat{g}$ found above, we find

$$
\delta W_{k}^{(n a)}=2 \int d^{3} \mathbf{x} d^{3} \mathbf{v} F E\left(\bar{\omega}-\omega_{*}\right) \sum_{l, l^{\prime}} \frac{J_{l} J_{l^{\prime}}^{*}}{\omega-k_{l}\left\langle v_{\|}\right\rangle} e^{i\left(l^{\prime}-l\right) \theta} .
$$

or

$$
\delta W_{k}^{(n a)}=2 \int d^{3} \mathbf{x} d^{3} \mathbf{v} F E\left(\bar{\omega}-\omega_{*}\right) \sum_{l} \frac{J_{l}^{2}}{\omega-k_{l}\left\langle v_{\|}\right\rangle} .
$$

Since

$$
P_{h}=\int E F d^{3} \mathbf{v}
$$

we also get

$$
\delta W_{k}^{(a)}=-\int d^{3} \mathbf{x} d^{3} \mathbf{v} E F \partial_{\psi_{p}} \log F\left(\kappa \cdot \xi_{\perp}^{*}\right)\left(\nabla \psi_{p} \cdot \xi_{\perp}\right)
$$

It is straightforward to verify that $\left|\delta W_{k}^{(a)}\right|,\left|\delta W_{k}^{(n a)}\right| \ll|\delta K|,\left|\delta W_{f}\right|$ if $\beta_{h} \ll a / R$ and $\rho \ll a$ which are true in general. Here $a$ and $R$ are the minor and major radii respectively, and $\rho$ is the particle gyro radius. We thus assume that the hot particles will not change the MHD eigenmode profile. The calculation of the eigenmode profile has been studied analytically ${ }^{6}$ and numerically. ${ }^{3}$ The eigenmode used in this paper is found using the NOVA-K code (See Fig. 1).

Write the perturbation in the following form:

$$
\delta \mathbf{B}_{\perp}=\nabla \times\left(\xi_{\perp} \times \mathbf{B}\right)=\nabla \times \alpha \mathbf{B} R_{0}
$$

with

$$
\alpha=\sum \alpha_{m n}\left(\psi_{p}\right) e^{i(n \phi-m \theta-\omega t)}
$$


we wish to express $\xi_{\perp}$ in terms of $\alpha$ since $\alpha$ gives the form of the perturbation used in the guiding-center equations.

Introduce the gauge function $G$ through

$$
\alpha \mathbf{B} R_{0}=\xi_{\perp} \times \mathbf{B}+i \nabla G
$$

We have

$$
\xi_{\perp}=i \frac{\nabla G \times \mathbf{B}}{B^{2}}
$$

and the Fourier component of $G$ is

$$
G_{m}\left(\psi_{p}\right)=\frac{I+g q}{m-n q} \alpha_{m}\left(\psi_{p}\right)
$$

thus for a given $\alpha$, we can readily find the following relations:

$$
\begin{gathered}
\xi_{\perp} \cdot \kappa=\frac{i}{B^{2} \mathcal{J}}\left\{\partial_{\theta} G \partial_{\psi_{p}} B-\partial_{\theta} B \partial_{\psi_{p}} G\right\} \\
\xi_{\perp} \cdot \nabla \psi_{p}=\frac{i}{B^{2} \mathcal{J}}\left\{g \partial_{\theta} G-I \partial_{\phi} G\right\}
\end{gathered}
$$

where $g=R B_{\phi}, I(\psi)$ is the toroidal crurrent inside the flux surface $\psi, q(\psi)$ is the safety factor, $\mathcal{J}$ is the Jacobian and $\mathcal{J} B^{2}=I+g q$.

We only consider a single low $n$ TAE mode with the two most important poloidal harmonics here, i.e. $n=1 ; m=1,2$. The sum in Eq. (25) then consists of four terms, with $l=m \pm 1$. The dispersion relation including hot particles is

$$
\frac{1}{2}\left(-\omega^{2}+\omega_{0}^{2}\right) \int \rho|\xi|^{2} d^{3} x+\delta W_{k}^{(a)}+\delta W_{k}^{(n a)}=0
$$

where $\omega_{0}=v_{A} /\left(2 q_{r} R\right)$ is the eigenmode frequency, $v_{A}$ is the Alfven velocity and $q_{r}=1.5$ for the mode chosen. One can solve this equation for the frequency using a complex root finder. However, it is equally accurate to find the roots using a perturbative method due to the small contribution from $\alpha$-particles. 
Let $\omega=\omega_{r}+i \omega_{i}, K=(1 / 2) \int \rho\left|\xi_{\perp}\right|^{2} d^{3} \mathbf{x}$. We then find

$$
\left\{\begin{array}{l}
\omega_{r}=\omega_{0}+\frac{1}{2 \omega_{0} K}\left[\delta W_{k}^{(a)}+\operatorname{Re}\left\{\delta W_{k}^{(n a)}\right\}\right] \\
\omega_{i}=\frac{1}{2 \omega_{0} K} \operatorname{Im}\left\{\delta W_{k}^{(n a)}\right\}
\end{array}\right.
$$

where $\omega_{0}$ could be either positive or negative. We are only interested in the positive $\omega_{i}$ here.

Assuming the fluid density profile is

$$
\rho\left(\psi_{p}\right)=\rho_{0} F_{i}\left(\psi_{p}\right)
$$

where $\rho_{0}$ is the central ion density and $F_{i}$ is the ion density distribution function, we find

$$
K=(1 / 2) \rho_{0} \int F_{i}\left(\psi_{p}\right)|\xi|^{2} \mathcal{J} d \psi_{p} d \theta d \phi
$$

For a given equilibrium and eigenmode, $K$ is a constant.

These solutions for $\omega_{r}, \omega_{i}$ are used to advance the mode using the instantaneous particle distribution.

\section{Numerical Simulation}

\section{A. Guiding Center Equations}

A Hamiltonian guiding center code ${ }^{10,11}$ ORBIT is used to evaluate $\delta W_{k}^{(a)}$ and $\delta W_{k}^{(n a)}$. Bounce averages are conveniently converted to time integrals.

The guiding-center Hamiltonian is

$$
\mathcal{H}=\frac{1}{2} \rho_{\|}^{2} B^{2}+\mu B+\Phi
$$

with four Hamiltonian variables

$$
\phi, \theta, P_{\phi}=g \rho_{i}-\psi_{p}, P_{\theta}=I \rho_{c}+\psi_{t}
$$


where $\rho_{c}=\rho_{\|}+\alpha, \rho_{\|}=v_{\|} / B, \psi_{t}$ is the toroidal flux with $d \psi_{t} / d \psi_{p}=q, \Phi$ is the electric potential and $\alpha$ gives the magnetic perturbation through $\delta \mathbf{B}_{\perp}=\nabla \times \alpha \mathbf{B}$. The guiding center equations are ${ }^{12,10}$

$$
\begin{array}{ll}
\dot{P}_{\phi}=-\partial_{\phi} \mathcal{H}, & \dot{\phi}=\partial_{P_{\phi}} \mathcal{H} \\
\dot{P}_{\theta}=-\partial_{\theta} \mathcal{H}, & \dot{\theta}=\partial_{P_{\theta}} \mathcal{H}
\end{array}
$$

It is also easy to find the radial drift

$$
\dot{\psi}_{p}=\frac{g}{D} \dot{P}_{\theta}-\frac{I}{D} \dot{P}_{\phi}
$$

and the parallel acceleration

$$
\dot{\rho}_{\|}=\frac{1}{g}\left(\dot{P}_{\theta}+\dot{\psi}_{p}\right)-\left(P_{\theta}+\psi_{p}\right) \frac{g^{\prime}}{g^{2}} \dot{\psi}_{p}-\dot{\alpha}
$$

where

$$
\dot{\alpha}=\dot{\theta} \partial_{\theta} \alpha+\dot{\psi}_{p} \partial_{\psi_{p}} \alpha+\dot{\phi} \partial_{\phi} \alpha+\partial_{t} \alpha
$$

and $D=g q+I+\rho_{\|}\left(g I^{\prime}-I g^{\prime}\right)$.

A Runge-Kutta fourth order integration scheme is then employed to solve the guiding center equations. Here and in the following we use units given by the on-axis gyro frequency (time), and the major radius (distance). Using the lowest order expression for $\dot{\theta}$ it is straightforward to convert a bounce average to a time average

$$
\langle f\rangle=\frac{1}{T} \int f d t
$$

with $\mathrm{T}$ a bounce (transit) period, or any period much longer than a bounce (transit) period. 


\section{B. Monte Carlo Particle Distribution}

Write the particle density in terms of the distribution function

$$
d N=F d^{3} \mathbf{x} d^{3} v
$$

and use the Monte-Carlo representation for the density (integrated over $\theta$ and $\phi$ )

$$
d N=c \sum_{k} \delta\left(\psi_{p}-\psi_{p k}\right) \delta\left(\mu-\mu_{k}\right) \delta\left(E-E_{k}\right) d \psi_{p} d \mu d E
$$

Upon normalizing $\mathrm{F}$ through $\beta_{h}$

$$
\beta_{h}=\frac{8 \pi}{B^{2} V} \int E d N
$$

we find the density element in the Monte-Carlo representation to be

$$
F d^{3} \mathrm{x} d^{3} \mathrm{v}=\frac{B^{2} V \beta_{h}}{8 \pi \sum_{j} E_{j}} \sum_{k} \delta\left(\psi_{p}-\psi_{p k}\right) \delta\left(\mu-\mu_{k}\right) \delta\left(E-E_{k}\right) d \psi_{p} d \mu d E
$$

where $V$ is the volume.

Fixed values of $\psi_{p}, \mu, E$ and sign $v_{\|}$completely define the orbits within bounce averaging, which is carried out numerically, i. e. particles are initiated at particular values of $\psi_{p}, \theta$ and $\phi$, and the orbits followed. Thus $F$ is the total particle density integrated over $\theta$ and $\phi$ at any time. This $\mathrm{F}$ may be regarded as a bounce-averaged distribution. Substituting $F$, we get

$$
\delta W_{k}^{(n a)}=\left.\frac{B^{2} V \beta_{h}}{4 \pi \sum_{j} E_{j}} \sum_{k} E_{k}\left(\omega_{*}-\bar{\omega}\right) \sum_{l} \frac{J_{l}^{2}}{k_{l}\left\langle v_{\|}\right\rangle-\omega}\right|_{k}
$$

and

$$
\delta W_{k}^{(a)}=-\left.\frac{B^{2} V \beta_{h}}{4 \pi \sum_{j} E_{j}} \sum_{k} E_{k} \partial_{\psi_{p}} \log F\left(\kappa \cdot \xi_{\perp}\right)\left(\nabla \psi_{p} \cdot \xi_{\perp}\right)\right|_{k}
$$

where $\left.\right|_{k}$ means the function is evaluated at $\psi_{p}=\psi_{p, k}, E=E_{k}, \theta=\theta_{k}, \ldots$, etc. 


\section{Evaluation of $\partial_{\psi_{p}} \log F$ and $\partial_{E} \log F$}

In order to calculate $\delta \hat{W}_{k}$ we need to know both $\partial_{E} \log F$ and $\partial_{\psi_{p}} \log F$. This cannot be done directly if each particle is represented as a point in phase space. Either phase space must be partitioned and local densities calculated, or equivalently each particle can be assigned a finite size in both real and velocity space.

Rewrite the Monte-Carlo distribution function in the form

$$
F=\sum_{k}^{N} \frac{1}{\left(\psi_{p}-\psi_{p k}\right)^{2}+a^{2}} \frac{1}{\left(E-E_{k}\right)^{2}+b^{2}}
$$

where $a$ and $b$ are the widths in real and energy space respectively. We ignore the normalization constant since we are only interested in $\partial_{E} \log F$ and $\partial_{\psi_{p}} \log F$. Partial derivatives with respect to $\psi_{p}$ and $E$ are given by

$$
\partial_{\psi^{\prime} p} F=\sum_{k}^{N} \frac{-2\left(\psi_{p}-\psi_{p k}\right)}{\left[\left(\psi_{p}-\psi_{p k}\right)^{2}+a^{2}\right]^{2}} \frac{1}{\left(E-E_{k}\right)^{2}+a^{2}}
$$

and

$$
\partial_{E} F=\sum_{k}^{N} \frac{-2\left(E-E_{k}\right)}{\left[\left(E-E_{k}\right)^{2}+b^{2}\right]^{2}} \frac{1}{\left(\psi_{p}-\psi_{p k}\right)^{2}+a^{2}}
$$

The evaluation of $\operatorname{Im} \delta W_{k}^{(n a)}$ is obtained by the same technique rather than by using a delta-function. This method gives a very accurate evaluation in test cases using known distribution functions. We typically use more than ten thousand particles in the simulations and choose $a, b$ so that the particle widths are $5 \%$ of the total flux and energy domains.

\section{Numerical Results}

We treat the background plasma with ideal MHD, with the reponse given by the dispersion relation. The hot component is directly simulated. Ten thousand particles are used in the following simulations. Each particle is advanced in time according to the guiding 
center equations. Once a particle hits the wall, it is removed. Although we neglect the finite Larmor radius, we keep all the particle dynamics, including the finite orbit width. In fact, the latter is much more important than the former since it can significantly modify the mode-particle coupling. ${ }^{4}$ The initial spatial distribution is Gaussian and we use a slowing down distribution in energy. The distribution is uniform in pitch angle $\lambda=v_{\|} / v$ and uniform in poloidal angle $\theta$.

We assume that the mode structure does not change except for the amplitude and phase as time evolves. This is a valid assumption because of the small contribution of the kinetic terms to the dispersion relation. The mode structure is fixed by MHD. The mode amplitude $A(t)$ is advanced in time according to the equation

$$
A(t+\Delta t)=A(t)[1+\gamma(t) \Delta t]
$$

where the mode growth rate $\gamma(t)=\omega_{i}$ is determined by the dispersion relation. Similarly the phase is advanced according to $\alpha \sim e^{-i z}$ with $z(t+\Delta t)=z(t)+\omega(t) \Delta t$. The time step $\Delta t$ must be chosen small enough so that $|\gamma| \Delta t \ll 1$ and $\omega \Delta t \ll 1$.

Background damping $\gamma_{d}$ due to Landau resonance, collisions and the continuum, can be simply included through

$$
\gamma(t)=\omega_{i}-\gamma_{d}
$$

We solve the dispersion relation every 10 transits (one transit $=2 \pi R / v$ ) which in our case is much longer than the bounce time but short enough so that the alpha distribution does not change much. The total run time is chosen so that the mode saturation is clearly seen. We have performed simulations with parameters typical for the Tokamak Fusion Test Reactor (TFTR) and for the International Thermonuclear Experimental Reactor (ITER). ${ }^{13}$ The initial radial width of the Gaussian alpha particle distribution is varied over a range from $1 / 5$ to $1 / 3$ of the minor radius. Other profile shapes also produce equivalent results. In the following simulations, we use the typical TFTR parameters: $R=250 \mathrm{~cm}, a / R=$ 
$0.3, B=5 T, \beta_{\alpha}=0.2 \%, \omega_{0} / \Omega_{c}=0.006$ and $\gamma_{d} / \omega_{0}=0.1 \%$. Here $\Omega_{c}$ is the alpha particle gyro frequency. The estimate for $\gamma_{d}$ includes electron- and ion-Landau damping, continuum damping and collisional damping ${ }^{14-16}$ and is conservatively small. For ITER, we use $R=$ $775 \mathrm{~cm}, a / R=0.36, B=6 T, \beta_{\alpha}=3 \%, \omega_{0} / \Omega_{c}=0.0014$ and $\gamma_{d}=0$. Thus for both devices we consider worst case scenarios, with damping much smaller than currently available estimates.

Figure 1 plots the safty factor $q$ and the poloidal harmonics of the eigenfunction $\xi_{r}$ of the $n=1 \mathrm{TAE}$ eigenmode versus minor radius $r$. Other poloidal harmonics of $\xi_{r}$ such as $m=0$ and $m \geq 4$ are not shown here since they are negligibly small.

From the numerical results the mode saturation mechanism and the particle loss mechanism are easily understood. First examine mode saturation. Fig. 2 and Fig. 3 plot the initial and the final particle density and energy distributions for the TFTR case. We show the number of particles in bins of equal spacing in $(r / R)^{2}$, since a real space volume element $d^{3} \mathbf{X} \sim d r^{2}$. Resonant particles which lose energy to the mode through inverse Landau damping shift outwards, thus the density gradient, which gives the free energy to the wave, decreases as time evolves and eventually the wave saturates. Also the particle energy gradient becomes steeper, since high energy particles lose energy whereas low energy particles do not interact with the mode. Thus the mode further loses drive due to the increased velocity space Landau damping and eventually saturates.

The flattening of the density profile due to mode resonance is difficult to see, because the resonance position depends on $v_{\|}$and thus is continuously distributed radially because of the distribution in pitch. Thus with a uniform pitch distribution the "flattening" consists of a general spreading of the distribution. To illustrate the numerical occurance of the resonance, we have nerformed a simulation with all the particles having the same energy $E=0.7 E_{\alpha}$, and all co-moving with pitch $v_{\|} / v=1$. We keep the mode amplitude fixed $\left(\delta B / B \sim 10^{-2}\right)$ and examine the density profile before and after a simulation lasting 200 
transit times. The analytic estimate of the resonant position is $r^{2} / R^{2}=0.01$ and we have used ITER parameters. The density flattening due to the Landau resonance is clearly seen in Fig. 4.

We find for ITER for a variety of initial profiles that the mode saturates before it becomes large enough to induce particle loss. The maximum linear growth rate is $0.2 \%$ of the mode frequency. In Fig. 5 are shown sample saturation results. The maximum value is $\delta B / B \sim 2 \times 10^{-4}$ and the total losses are unobservable. As in the case of the fishbone, the mode produces additional profile modification before it can decay away, resulting in a strong decay rate. The time scale for the profile modification by the mode is orders of magnitude shorter than the time scale for alpha particle replenishment, so the additional source of new alpha particles would not modify this result. Furthermore, the time scale is much shorter than the collision time so that the conversion between trapped and passing particles is negligible during the profile modification.

For TFTR with additional damping $\gamma_{d} / \omega_{0}=.1 \%$, the mode continues to grow until some alpha particle loss (less than $2 \%$ ) is produced. The linear growth rate is $0.1 \%$ of the mode frequency. Estimates of $\gamma_{d}$ are larger than this, so unless very special low damping conditions are produced, we expect the mode to be stable.

The quasilinear stabilization results can be understood from a simple analysis. From Eq. (36) the growth rate $\gamma$ is proportional to $\left(\omega_{*}-\bar{\omega}\right)$. Using the approximate form of the distribution function $F \sim E^{-3 / 2} e^{-\left(r / L_{n}\right)^{2}}$, we find $\gamma \sim\left[\left(a / L_{n}\right)^{2}-\Delta^{2}\right]$ with $\Delta=$ $\sqrt{3 m_{\alpha} \Omega_{c} \omega a^{2} /(4 E)}$. From $E=0.5 m_{\alpha} \omega^{2} / k_{\|}^{2}$ with $k_{\|} \simeq(n-m / q) / R$, we find $\Delta=3.9$ for ITER, and $\Delta=1.6$ for TFTR. In ITER very modest profile changes can lead to stabilization, as is clear from Fig. 5. However, without additional collisional or continuum damping, mode stabilization in TFTR does not occur until the alpha profile has a width comparable to the minor radius or until the energy gradient has been steepened to balance the drive. 
Now examine particle loss. It is interesting to analyze the loss mechanism, as high energy particles produced by ion cyclotron heating or neutral beam injection can produce more strongly unstable cases than the alpha driven cases discussed here. The final particle distribution in poloidal angle $\theta$ is shown in Fig. 6 . The bump around $\theta=0$ comes from the co-moving particles since their orbits are shifted outward and they thus spend more time on the low field side. It also includes those particles originally near the passing/trapped boundary which become trapped due to their energy loss to the wave. The bump near $\theta=\pi$ is due to the counter-passing particles since their orbits are shifted inward and therefore they spend more time on the high field side.

Figure 7 plots the final particle pitch $\lambda=v_{\|} / v$ and the lost particle pitch distributions. The bump in the confined particle distribution is due to those trapped particles near the passing/trapped boundary. The hole on the other hand, is due to both the initial prompt loss (barely trapped large banana width particles) and to the loss of barely counter-passing particles which lose energy to the wave, transfer into a fat banana orbit, and then hit the wall in the co-moving direction. Mathematically, since $\lambda=v_{\|} / v=\sqrt{1-\mu B / E}$, we have

$$
\frac{d \lambda}{d E} \sim \frac{1}{\sqrt{1-\mu B / E}}
$$

that is, for the same energy loss, those particles trapped or near the trapped/passing boundary will have the largest change in pitch angle $\lambda$. The lost particle pitch distribution is due to the initial prompt loss and barely trapped/passing particles. It is easy to verify that a lost barely trapped particle has a average pitch $\lambda \simeq \sqrt{1-B_{\min } / B_{\max }} \simeq \sqrt{2 r} \simeq \sqrt{\varepsilon}=0.6$ for our parameters, which is in good agreement with the simulation. This result as well as the total observed number of lost particles also agrees with a previous study ${ }^{1}$ of TAE induced loss, which however did not include a self corsistent calculation of the mode stabilization. Note that as soon as particles become trapped the resonance with the mode becomes very weak. This contrasts significantly with the fishbone case, where a trapped particle can remain in resonance as it is moved radially outwards to the wall. 
Figures 8 and 9 show the mode saturation and induced particle loss respectively. The saturation mechanis $\mathrm{n}$ includes the density flattening and energy gradient steepening. The mode saturates before it becomes large enough to induce stochasticity in the $\alpha$-particle orbits. The final value is $\delta B / B \sim 3 \times 10^{-3}$. The time scale for particle distribution modification is short compared to alpha particle replenishment and collision times. The dominant loss mechanism is that of barely counter-passing particles losing energy to the wave, transfering into fat banana orbits, and then hitting the outside wall in the co-moving direction. Most particles transfer to trapped orbits and intersect the wall while co-moving, but a few shift outward far enough to intersect the wall as counter moving passing particles. The losses are thus very effective for large banana width. In TFTR all such barely trapped particles are lost due to their relative large banana width whereas in ITER they remain in the device and contribute to mode damping.

With strong mode destabilization such as might be provided by neutral beam injection, large losses would occur in TFTR and should lead to "explosive pulsations". 7 A more extensive analysis of such a case, including lost particle deposition profiles, etc. will be examined in a future publication. But expected alpha particle densities are not sufficient to destabilize the mode in the presence of estimated damping. In the alpha particle driven case in ITER even without additional damping the particle response is local and sufficient to modify the local gradients, thereby stabilizing the mode, but not sufficient to cause losses. The possibility of several $\mathrm{n}$ values simultaneously unstable will be considered in a future publication, but the loss mechanism is weak enough that we do not expect serious losses due to alpha particles even in this case.

Our results are similar to the partial results obtained by using the three-dimensional hybrid gyrokinetic-MHD code ${ }^{17}$ MH3D in which at least 200 thousand particles were necessary to overcome the random noise (local particle effects influence the fluid elements).

It is also worth pointing out that if the hot particles are produced by a counter-parallel 
injected beam, then particles are lost through their losing energy to the wave, shifting outwards and thereby executing larger drift excursions as a consequency of being closer to the trapped/passing boundary, eventually hitting the wall in the counter-moving direction.

The mode frequency and the growth rate are depicted in Fig. 10 and Fig. 11 respectively. The mode frequency is very close to the TAE mode frequency $\omega_{0}$ while the growth rate is very small compared with $\omega_{0}$, thus the perturbative method used is a very reasonable approximation. The mode growth rate obtained in this work agrees with that given by the NOVA-K code. ${ }^{3}$

\section{Conclusion}

The interaction of a single toroidal mode number $n=1$ Alfven eigenmode with energetic alpha-particles is investigated self-consistently by using a realistic dispersion relation. We retain all important poloidal mode numbers and their radial mode profiles calculated with the NOVA-K code. A Hamiltonian guiding center code is used to provide a fast and accurate way to calculate the bounce averaged expressions entering in the dispersion relation. The study includes the particle finite banana orbit width, all toroidal particle dynamics and the mode-particle interaction. We have used an analytic circular flux surface equilibrium, but the code can also use numerical equilibria including effects due to plasma shape, Shafranov shift, and plasma rotation. Mode saturation is observed through the flattening of the real space density gradient and the steepening of the energy gradient. Total losses are small in TFTR and zero in ITER, and the mode amplitude saturates at a level which is too small to induce stochastic loss. We do not expect these results to be changed by the effects of magnetic well and plasma shape, as they have little effect on transit frequency phenomena. 


\section{ACKNOWLEGMENTS}

We are grateful to Drs. C. Z. Cheng and G. Y. Fu for useful discussions. This work was supported by the U.S. Department of Energy under contract number DE-AC02-76CHO3073. 


\section{References}

${ }^{1}$ D. J. Sigmar, C. T. Hsu, R. B. White, and C. Z. Cheng, Phys. Fluids B 4 (6), 1506 (1992).

${ }^{2}$ C. Z. Cheng, Phys. Fluids B 2, 1427 (1990).

${ }^{3}$ C. Z. Cheng, Phys. Reports 211, 1 (1992).

${ }^{4}$ G. Y. Fu, C. Z. Cheng, and K. L. Wong, Phys. Fluids B 5, 4040 (1993).

${ }^{5}$ G. Y. Fu and C. Z. Cheng, Phys. Fluids B 4, 3722 (1992).

${ }^{6}$ C. Z. Cheng and M. S. Chance, Phys. Fluids 29, 3695 (1986).

${ }^{7}$ B. N. Breizman, H. L. Berk, and H. Ye, Phys. Fluids B 5, 3217 (1993).

${ }^{8}$ J. T. M. Antonsen and B. Lane, Phys. Fluids 23, 1205 (1980).

${ }^{9}$ P. J. Catto, W. M. Tang, and D. E. Baldwin, Plasma Phys. 23, 639 (1981).

${ }^{10}$ R. B. White and M. S. Chance, Phys. Fluids 27, 2455 (1984).

${ }^{11} \mathrm{R}$. B. White and Y. Wu, Numerical evaluation of high energy particle effects in magnetohydrodynamics, To appear in Phys. Fluid B.

${ }^{12}$ R. B. White, Phys. Fluids B 2, 845 (1990).

${ }^{13} \mathrm{~J}$. Wesson, Tokamaks, Oxford Science Publications, 1987.

${ }^{14} \mathrm{~F}$. Zonca and L. Chen, Phys. Rev. Lett. 68, 592 (1992).

${ }^{15}$ M. N. Rosenbluth, H. L. Berk, J. W. VanDam, and D. M. Lindberg, Phys. Rev. Lett. 68, 596 (1992). 
${ }^{16}$ C. Z. Cheng, G. Y. Fu, H. E. Mynick, R. V. Budny, R. B. White, S. J. Zweben, C. T. Hsu, D. J. Sigmar, D. A. Spong, B. A. Carreras, and C. L. Hedrick, in Proceedings of the Fourteenth International Conference on Plasma Physics and Controlled Nuclear Fusion Research, IAEA, volume 2, p. 51, Vienna, 1993.

${ }^{17}$ G. Y. Fu and W. Park, Bull. Am. Soc. 38, 1945 (1993). 


\section{FIGURE CAPTIONS}

Fig. 1 The poloidal harmonics of eigenfunction $\xi_{r}$ of the $n=1$ TAE eigenmode versus $r$

Fig. 2 The initial and final density distribution for TFTR

Fig. 3 The initial and final energy distribution for TFTR.

Fig. 4 The density flattening due to a local Landau resonance.

Fig. 5 Mode amplitude versus time for ITEK with a $3 \%$ alpha particle beta. Three different distribution widths $L_{\alpha}$ are shown.

Fig. 6 Final theta distribution for TFTR.

Fig. 7 The final (a) and the lost (b) particle pitch angle distributions for TFTR.

Fig. 8 The evolution of the $m=1$ amplitude for TFTR.

Fig. 9 The prompt loss (near $t=0$ ) and induced particle loss versus time for TFTR.

Fig. 10 Real mode frequency versus time for TFTR.

Fig. 11 Mode growth rate versus time for TFTR. 


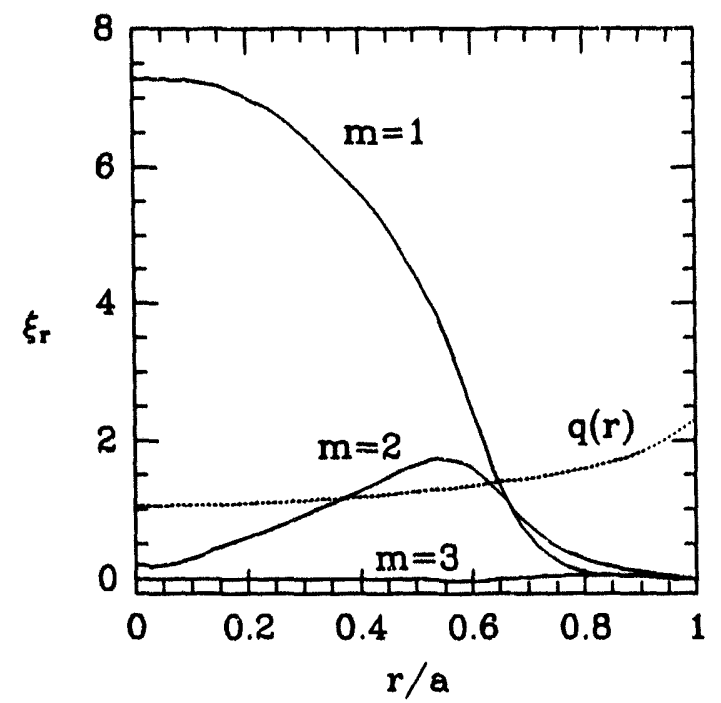

Figure 1: The poloidal harmonics of eigenfunction $\xi_{r}$ of the $n=1$ TAE eigenmode versus $r$.

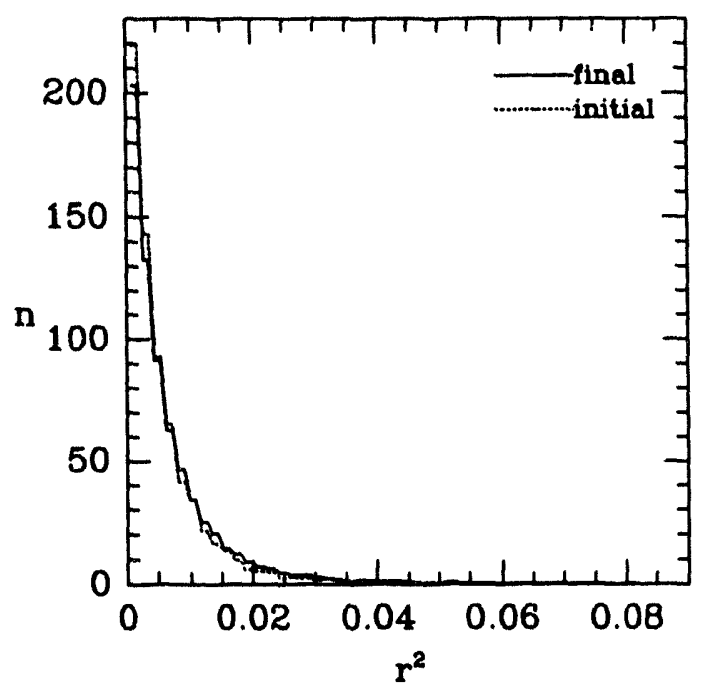

Figure 2: The initial and final density distribution for TFTR. 


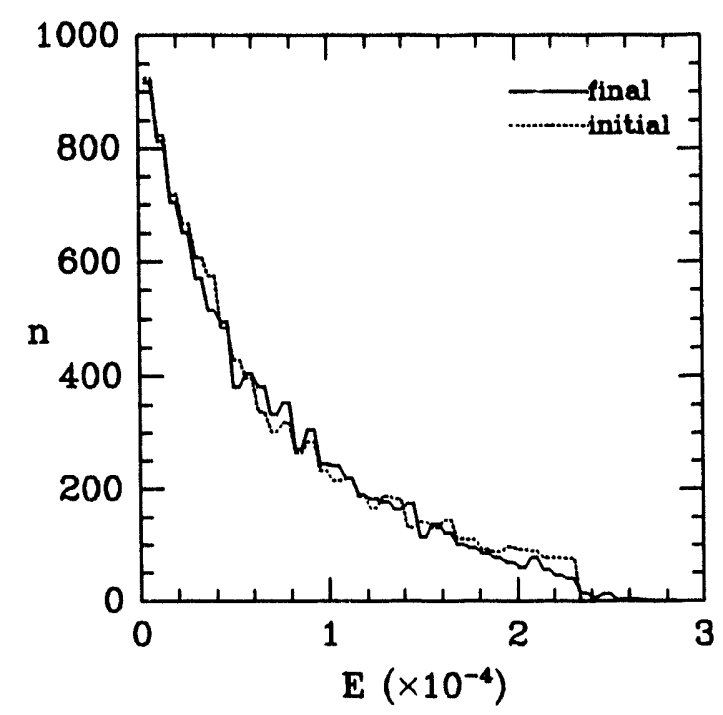

Figure 3: The initial and final energy distribution for TFTR.

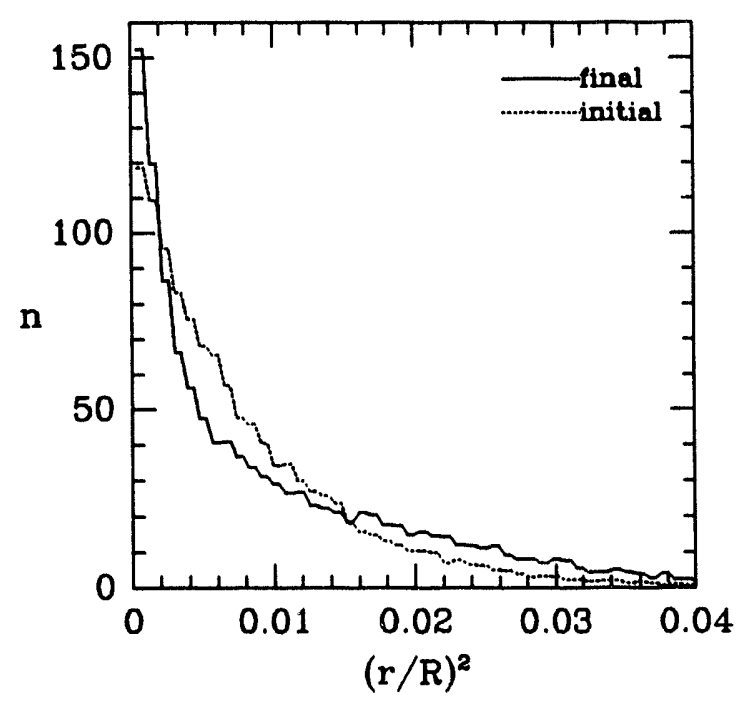

Figure 4: The density flattening due to a local Landau resonance. 


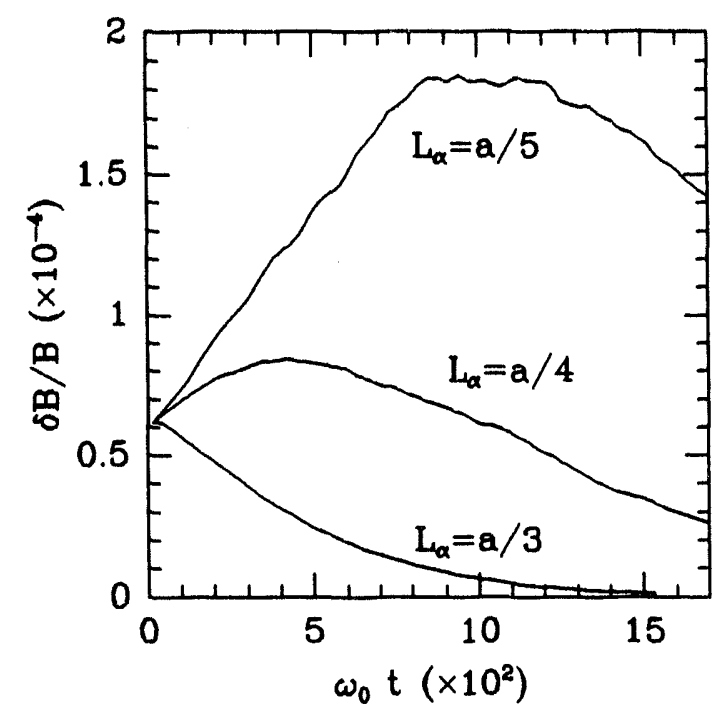

Figure 5: Mode amplitude versus time for ITER with a 3\% alpha particle beta. Three different distribution widths $L_{\alpha}$ are shown.

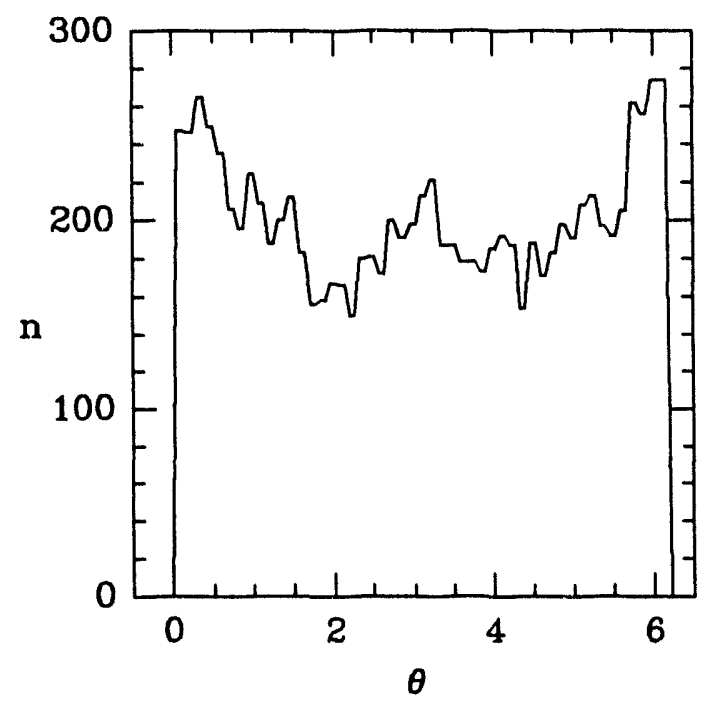

Figure 6: Final theta distribution for TFTR. 

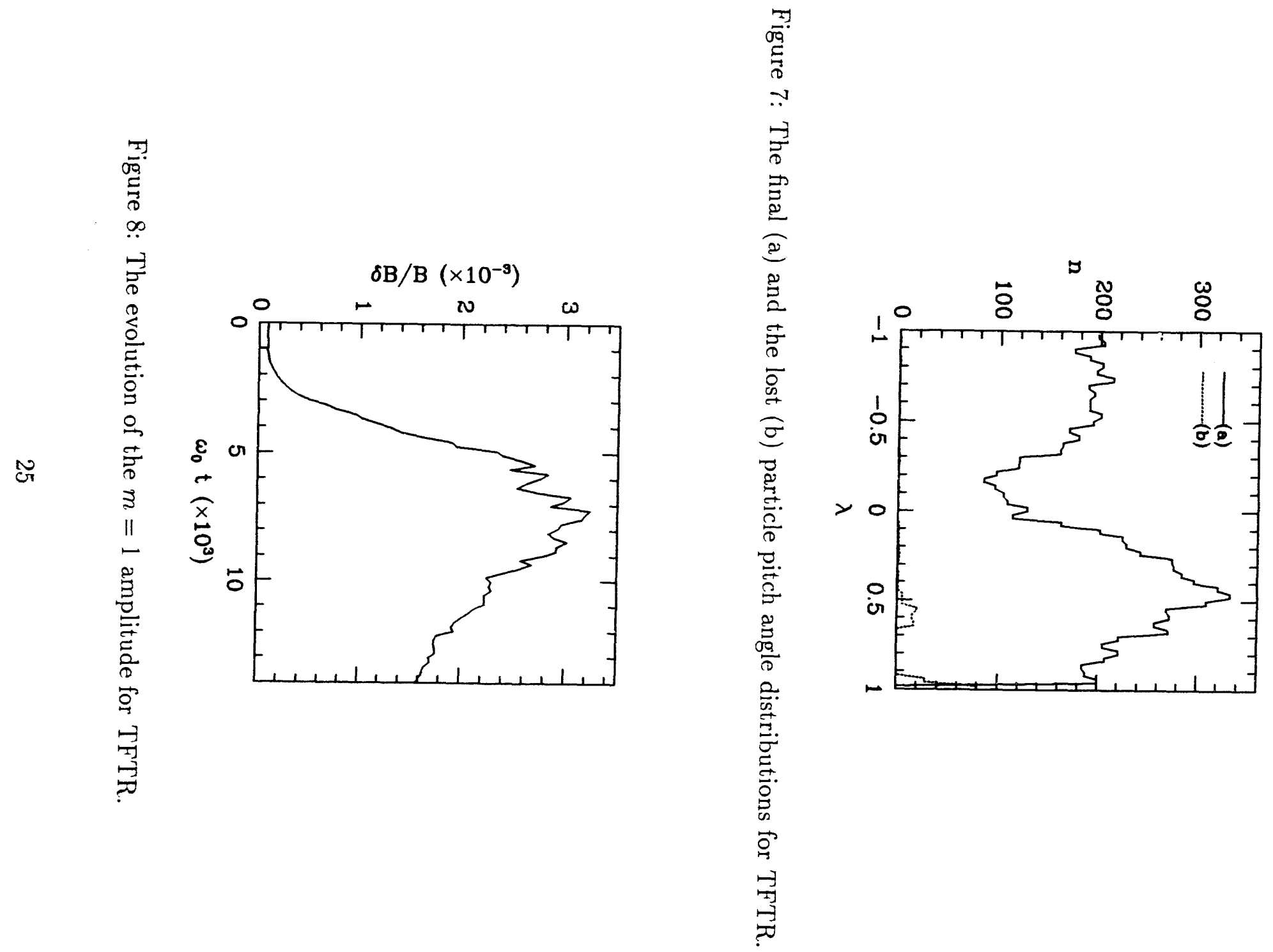


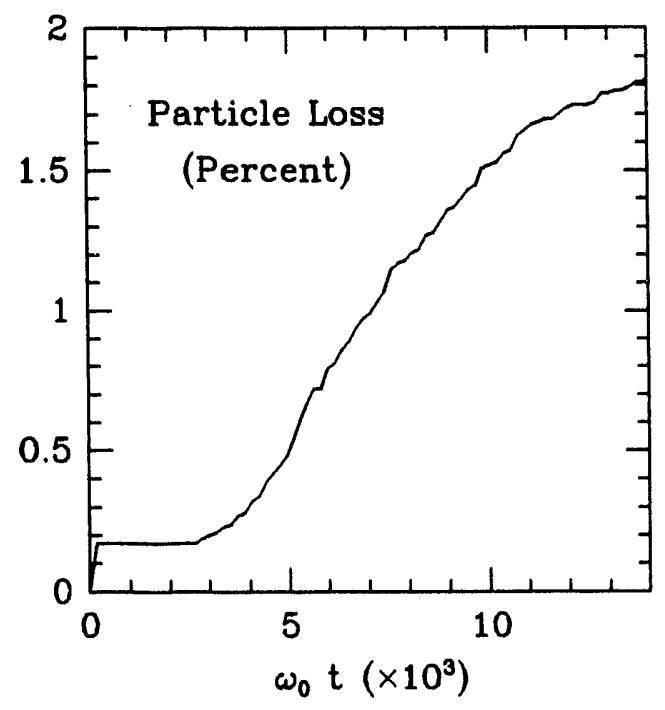

Figure 9: The prompt loss (near $t=0$ ) and induced particle loss versus time for TFTR.

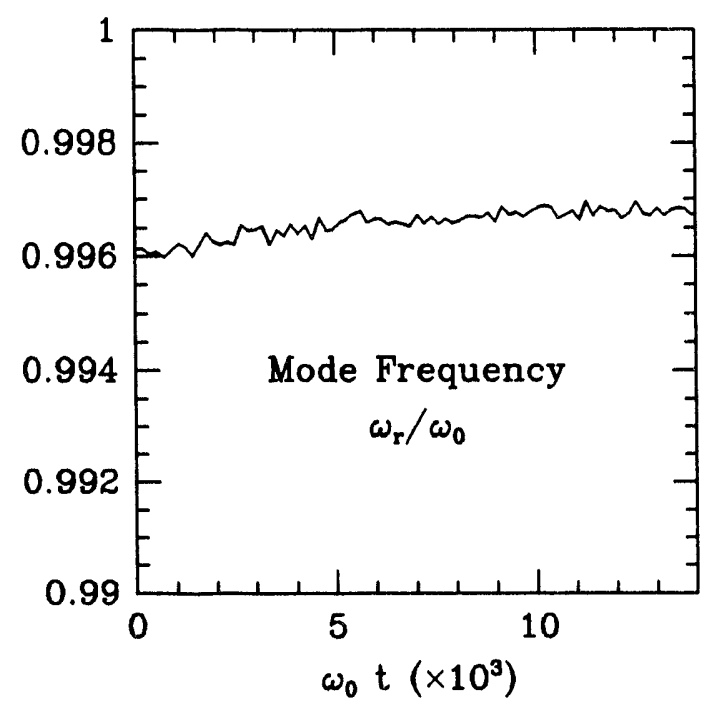

Figure 10: Real mode frequency versus time for TFTR. 


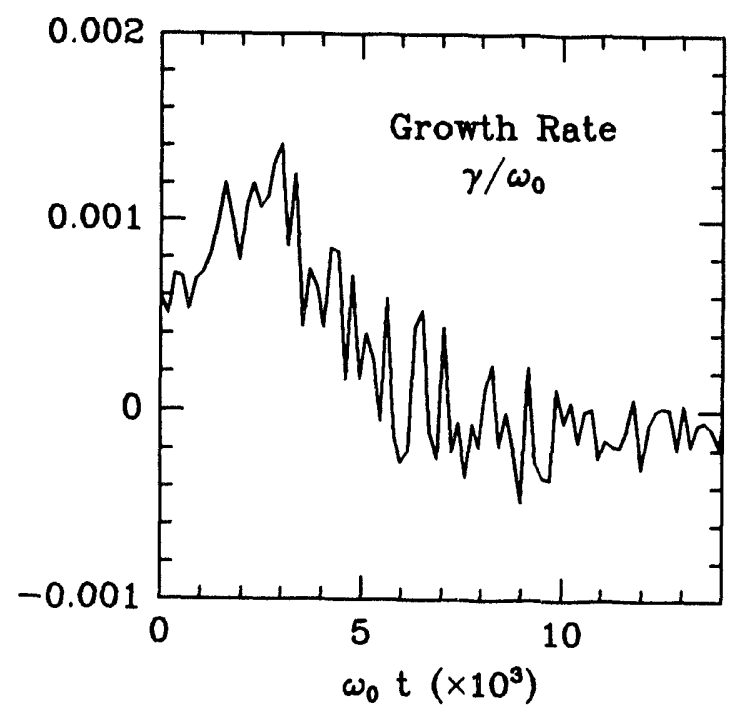

Figure 11: Mode growth rate versus time for TFTR. 
Dr. F. Paoloni, Univ. of Wollongong, AUSTRALIA

Prof. M.H. Brennan, Univ. of Sydney, AUSTRALIA

Plasma Research Lab., Australian Nat. Univ., AUSTRALIA

Prof. I.R. Jones, Flinders Univ, AUSTRALIA

Prof. F. Cap, Inst. for Theoretical Physics, AUSTRIA

Prof. M. Heindler, Institut für Theoretische Physik, AUSTRIA

Prof. M. Goossens, Astronomisch Instituut, BELGIUM

Ecole Royale Militaire, Lab. de Phy. Plasmas, BELGIUM

Commission-European, DG. XII-Fusion Prog., BELGIUM

Prof. R. Bouciqué, Rijksuniversiteit Gent, BELGIUM

Dr. P.H. Sakanaka, Instituto Fisica, BRAZIL

Prof. Dr. I.C. Nascimento, Instituto Fisica, Sao Paulo, BRAZIL Instituto Nacional De Pesquisas Espaciais-INPE, BRAZIL

Documents Office. Atomic Energy of Canada Ltd., CANADA

Ms. M. Morin, CCFMTokamak de Varennes, CANADA

Dr. M.P. Bachynski, MPB Technologies, Inc., CANADA

Dr. H.M. Skarsgard, Univ. of Saskatchewan, CANADA

Prof. J. Teichmann, Univ. of Montreal, CANADA

Prof. S.R. Sreenivasan, Univ. of Calgary, CANADA

Prof. T.W. Johnston, INRS-Energie, CANADA

Dr. R. Bolton, Centre canadien de fusion magnétique, CANADA

Dr. C.R. James, Univ. of Alberta, CANADA

Dr. P. Lukác, Komenského Universzita, CZECHO-SLOVAKIA

The Librarian, Culham Laboratory, ENGLAND

Library, R61, Rutherford Appleton Laboratory, ENGLAND

Mrs. S.A. Hutchinson, JET Library, ENGLAND

Dr. S.C. Sharma, Univ. of South Pacific, FIJI ISLANDS

P. Mähönen, Univ. of Helsinki, FINLAND

Prof. M.N. Bussac, Ecole Polytechnique, FRANCE

C. Mouttet, Lab. de Physique des Milieux lonisés, FRANCE

J. Fadet, CEN/CADARACHE - Bat 506, FRANCE

Prof. E. Economou, Univ. of Crete, GREECE

Ms. C. Rinni, Univ. of loannina, GREECE

Preprint Library, Hungarian Academy of Sci., HUNGARY

Dr. B. DasGupta, Saha Inst. of Nuclear Physics, INDIA

Dr. P. Kaw, Inst. for Plasma Research, INDIA

Dr. P. Rosenau, Israel Inst. of Technology, ISRAEL

Librarian, Intemational Center for Theo Physics, ITALY

Miss C. De Palo, Associazione EURATOM-ENEA, ITALY

Dr. G. Grosso, Istituto di Fisica del Plasma, ITALY

Prof. G. Rostangni, Istituto Gas lonizzati Del Cnr, ITALY
Dr. H. Yamato, Toshiba Res \& Devel Center, JAPAN

Prof. I. Kawakami, Hiroshima Univ., JAPAN

Prof. K. Nishikawa, Hiroshima Univ., JAPAN

Librarian, Naka Fusion Research Establishment, JAERI, JAPAN

Director, Japan Atomic Energy Research Inst., JAPAN

Prof. S. Itoh, Kyushu Univ., JAPAN

Research Info. Ctr., National Instit. for Fusion Science, JAPAN

Prof. S. Tanaka, Kyoto Univ., JAPAN

Library, Kyoto Univ., JAPAN

Prof. N. Inoue, Univ. of Tokyo, JAPAN

Secretary, Plasma Section, Electrotechnical Lab., JAPAN

S. Mori, Technical Advisor, JAERI, JAPAN

Dr. O. Mitarai, Kumamoto Inst. of Technology, JAPAN

Dr. G.S. Lee, Korea Basic Sci. Ctr., KOREA

J. Hyeon-Sook, Korea Alomic Energy Research Inst., KOREA

D.I. Choi, The Korea Adv. Inst. of Sci. \& Tech., KOREA

Prof. B.S. Liley, Univ. of Waikato, NEW ZEALAND

Inst of Physics, Chinese Acad Sci PEOPLE'S REP. OF CHINA

Library, Inst. of Plasma Physics, PEOPLE'S REP. OF CHINA

Tsinghua Univ. Library, PEOPLE'S REPUBLIC OF CHINA

Z. Li, S.W. Inst Physics, PEOPLE'S REPUBLIC OF CHINA

Prof. J.A.C. Cabral, Instituto Superior Tecnico, PORTUGAL

Prof. M.A. Hellberg, Univ. of Natal, S. AFRICA

Prot. D.E. Kim, Pohang Inst. of Sci. \& Tech., SO. KOREA

Prot. C.I.E.M.A.T, Fusion Division Library, SPAIN

Dr. L. Stenflo, Univ. of UMEA, SWEDEN

Library, Royal Inst. of Technology, SWEDEN

Prot. H. Wilhelmson, Chalmers Univ. of Tech., SWEDEN

Centre Phys. Des Plasmas, Ecole Polytech, SWITZERLAND

Bibliotheek, Inst. Voor Plasma-Fysica, THE NETHERLANDS

Asst. Prof. Dr. S. Cakir, Middle East Tech. Univ., TURKEY

Dr. V.A. Glukhikh,Sci. Res. Inst. Electrophys.I Apparatus, USSR

Dr. D.D. Ryutov, Siberian Branch of Academy of Sci., USSR

Dr. G.A. Eliseev, I.V. Kurchatov Inst., USSR

Librarian, The Ukr.SSR Academy of Sciences, USSR

Dr. L.M. Kovrizhnykh, Inst. of General Physics, USSR

Kemforschungsanlage GmbH, Zentralbibliothek, W. GERMANY

Bibliothek, Inst. Für Plasmaforschung. W. GERMANY

Prot. K. Schindler, Ruhr-Universitát Bochum, W. GERMANY

Dr. F. Wagner, (ASDEX), Max-Planck-Institut, W. GERMANY

Librarian, Max-Planck-Institut, W. GERMANY 

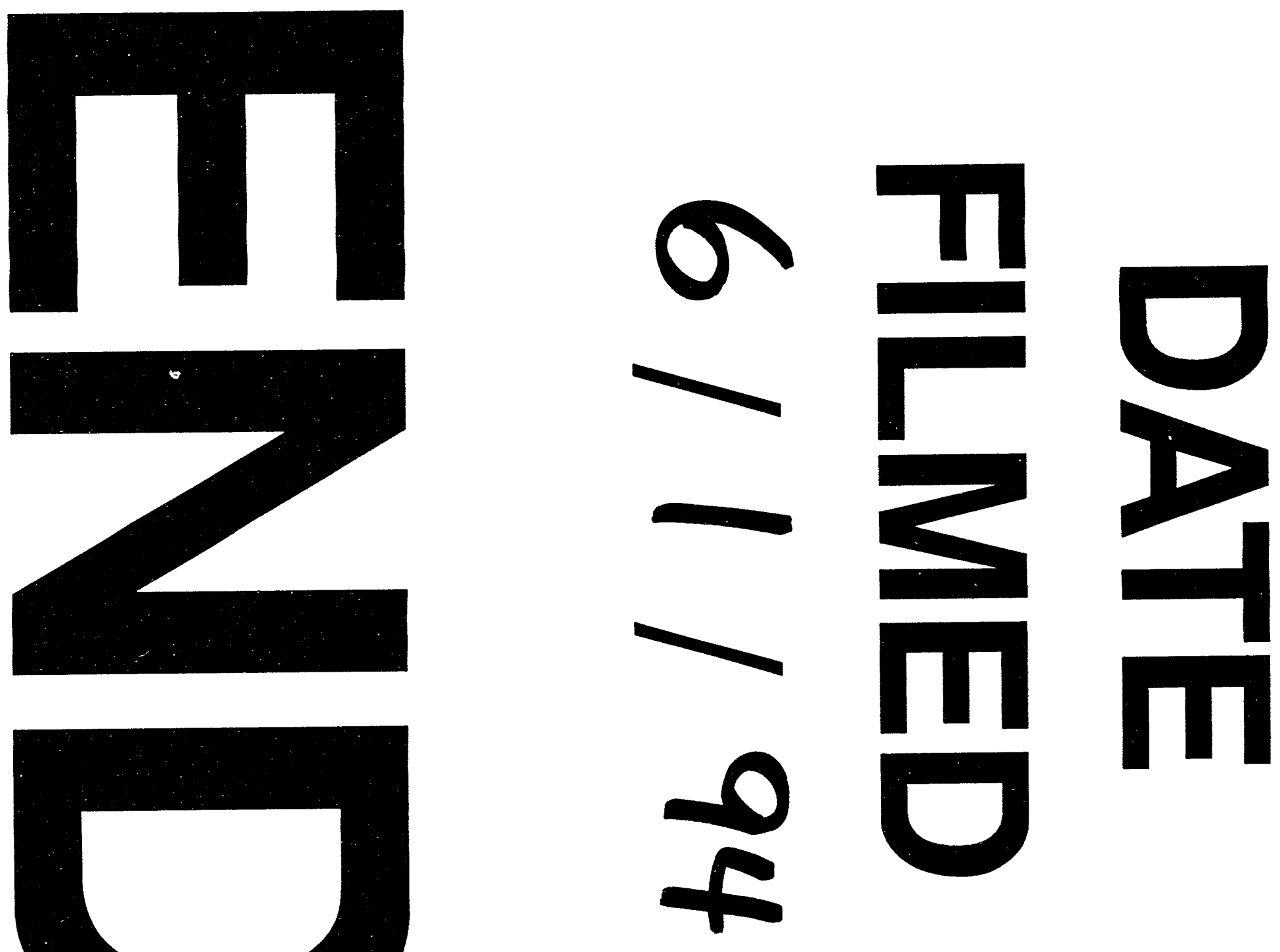
